\title{
Long-Horizon Exchange Rate Predictability?
}

Jeremy Berkowitz
Federal Reserve Board and Lorenzo Giorgianni

International Monetary Fund

January 1996

This Draft: September 19, 1996

\begin{abstract}
Several authors have recently investigated the predictability of exchange rates by fitting a sequence of long-horizon error-correction regressions. We show that in small to medium samples such a procedure gives rise to spurious evidence of predictive power. A simulation study demonstrates that even when using this technique on two independent series, estimates and diagnostic statistics suggest a high degree of predictability of the dependent variable. We apply a simple modification of the long-horizon regression due to Jegadeesh (1991), which may provide more accurate inference for researchers interested in comparing short and long-run predictability of U.S. dollar exchange rates.
\end{abstract}

Acknowledgements: The views presented are solely those of the authors and do not necessarily represent those of the Federal Reserve Board, the International Monetary Fund or their staff. Any remaining errors are ours. Correspondence to: Lorenzo Giorgianni, \#IS121286, International Monetary Fund, 700 19th Street, N.W., Washington, DC 20431, e-mail: 1giorgianni@imf.org. 


\section{Introduction}

The importance to applied economists of having statistical tools which can reliably test for the presence of long-run predictability in time series data can hardly be overstated. One such technique has gained prominence in recent years because of its apparent success in uncovering long-run relationships in international financial data. In particular, the approach commonly referred to as long-horizon regressions has been applied to the well known problem of predicting exchange rate movements by Mark (1995), Chinn and Meese (1995) and Bauer (1995). Earlier applications of this methodology include the study of equity return predictability (e.g., Fama and French (1988) and Campbell and Shiller (1988)).

In this comment, we explore the conjecture that much of the evidence of long-run exchange rate predictability may be an artifact of the statistical technique. We show that, for two independent time series, long-horizon regression are 'close' to spurious regressions. As a result, the finding of increasingly strong relationships at long horizons may not be evidence of an economic relationship.

We conduct several simulation experiments in which we generate two independent time series, one modeled after quarterly exchange rates and the other after the monetary fundamental as in Mark (1995) and Chinn and Meese (1995). The results confirm that several diagnostic statistics, such as t-statistics and $\mathbf{R}^{2}$, are increasingly biased away from zero for longer horizons of interest, casting doubt on the reliability of previous findings. Further, we show that application of an alternative regression procedure due to Jegadeesh (1991) yields inference at odds with the view that exchange rates are predictable at longer horizons.

The remainder of this comment proceeds as follows. In section 2, we describe longhorizon regressions as commonly applied to monetary models of exchange rate dynamics. In section 3, we describe the results of the Monte Carlo experiments. Section 4 introduces a modification of the standard long-horizon regression due to Jegadeesh (1991). Section 5 presents the estimation results using four leading dollar exchange rates. Section 6 concludes. 


\section{Long-Horizon Regressions}

The long-horizon regression approach entails estimating $\mathrm{k}$ individual equations,

$$
\Delta \mathrm{s}_{\mathrm{t}+\mathrm{k}}^{\mathrm{k}}=\alpha_{\mathrm{k}}+\beta_{\mathrm{k} \text { t }} \mathrm{z}_{\mathrm{k}, \mathrm{t}}
$$

where $s_{t}$ and $z_{t}$ are observed data, $\Delta^{k}$ is the $k^{\text {th }}$ difference operator and $\alpha_{k}$ and $\beta_{k}$ are the parameters to be estimated. If the $\beta_{k}$ 's, the associated t-statistics and the regression $R_{k}^{2}$ 's are found to increase with $\mathrm{k}$, the researcher takes this as evidence that $\mathrm{z}_{\mathrm{t}}$ can predict long-run changes in $s_{t}$ better than short-run movements.

In the context of monetary models of exchange rate dynamics, Mark (1995) proposes estimation of:

$$
\Delta \mathrm{s}_{\mathrm{t}+\mathrm{k}}=\alpha_{\mathrm{k}}+\beta_{\mathrm{k}}\left(\mathrm{f}_{\mathrm{t}}-\mathrm{s}_{\mathrm{t}}\right)+\epsilon_{\mathrm{k}, \mathrm{t}}
$$

where $\mathrm{f}_{t}=\left(\mathrm{m}_{\mathrm{t}}-\mathrm{m}_{\mathrm{t}}^{*}\right)-\left(\mathrm{y}_{\mathrm{t}}-\mathrm{y}_{\mathrm{t}}^{*}\right)$, with $\mathrm{m}_{\mathrm{t}}$ and $\mathrm{y}_{\mathrm{t}}$ denoting the $\log$ of M1 and of real GDP and asterisks representing foreign quantities.

This error-correction representation is motivated, on the one hand, by a large body of evidence which suggests that exchange rates and fundamentals may contain unit roots (e.g., Diebold (1988), Meese and Rose (1991)). Equation (2), at the same time, reflects a widely held view that exchange rates cannot move independently of macroeconomic fundamentals over long time horizons.

In the following discussion, we indicate various difficulties that may be associated with inference from equation (2), especially in presence of small samples and large k's.

First, specification (2) requires that the nominal exchange rate and the fundamental ("equilibrium" exchange rate) cointegrate, i.e., there exists a linear combination of these series which is stationary. Further, specification (2) requires that $s_{t}$ and $f_{t}$ cointegrate with cointegrating vector equal to [1 -1].

However, existing literature suggests very little evidence of cointegration between exchange rates and fundamentals. Mark (1995) and Chinn and Meese (1995), for example, do not find evidence of cointegration for several leading exchange rates. MacDonald and Marsh 
(1995) cannot reject cointegration, but reject a cointegrating vector of $[1-1] .{ }^{1}$ If the errorcorrection term is nonstationary, then adoption of specification (2) is inappropriate.

Second, the right-hand-side variable in equation (2) is predetermined but not exogenous and is highly positively autocorrelated. In this case the least squares (LS) estimate of the slope parameter, $\hat{\beta}_{\mathrm{k}}$, is biased away from zero in small samples. ${ }^{2}$ Mark (1995) attempts to correct for the bias in $\hat{\beta}_{\mathrm{k}}$. However, the bias in $\hat{\beta}_{\mathrm{k}}$ may, in turn, also lead to severe bias in other statistics associated with equation (2), such as t-statistics. This occurs because both the numerator and the denominator, the standard error of the regression, depend on $\hat{\beta}_{k}$.

Third, for $\mathrm{k}>1$, that is, when the forecasting horizon is greater than the sampling frequency, the error term of equation (2) will be a moving average process of order k-1, even under the null. To see this, note that if the exchange rate is well approximated by a random walk, then we may write $s_{t}=\sum_{i=1}^{t} \eta_{i}$, where $\eta_{i} \sim \mathrm{WN}\left(0, \sigma_{\eta}^{2}\right)$. So that equation (2) becomes

$$
\sum_{i=t+1}^{t+k} \eta_{i}=\alpha_{k}+\beta_{k}\left(f_{t}-s_{t}\right)+\epsilon_{k, t}
$$

It is well known that the presence of serial correlation in the residuals produces inconsistent LS standard errors of the coefficients.

Moreover, for very large $\mathrm{k}$, the dependent variable is itself approximately a random walk. So that, even if there is no statistical relationship between $s_{t}$ and $f_{t}$, the long-horizon regressions become close to a classical spurious regression.

In finite samples, one might reasonably expect larger $\mathrm{k}$ to be associated with larger t-statistics and $R^{2}$, even if $s_{t}$ is independent of $f_{t}$. If this is the case, doubt is cast upon the interpretability of the results of this methodology.

Mark undertakes to tabulate Monte Carlo (bootstrap) corrected critical values in order to account for the finite sample biases associated with equation (2). The bootstrap data are

\footnotetext{
${ }^{1}$ These results are not sensitive to the definition of the "fundamental" exchange rate implemented in various studies.
}

${ }^{2}$ See Mankiw and Shapiro (1986), Stambaugh (1986) and Nelson and Kim (1993). 
generated by fitting a restricted VAR to the error-correction term and changes in the exchange rate, $\left(\mathrm{z}_{\mathrm{t}}, \Delta \mathrm{s}_{\mathrm{t}}\right)$. Unfortunately, this bootstrap is likely to be invalid. If the exchange rate and fundamentals are independent then the error-correction term has a unit root. The standard bootstrap algorithm fails if the true process has a unit root, as shown by Basawa, Mallik, McCormick, Reeves and Taylor (1991) and Datta (1995). The design of this bootstrap is, in any event, inconsistent with the hypothesis that fundamentals and exchange rates are statistically unrelated.

\section{Simulation Experiment}

We emphasize that the appropriate null hypothesis associated with estimation of equation (2) is the independence of the two time series. Our simulation study, thus, investigates the results of estimating error-correction models with series which fail to cointegrate. This experimental design is supported by existing empirical evidence and properly reflects the null hypothesis contained in Mark (1995).

We generate independent Gaussian random variables, $s_{t}$ and $f_{t}$. The relative variances of the innovations of the two processes are calibrated to the estimated relative variances of the quarterly U.S. dollar/Deutsche mark (DM) and the monetary fundamental from 1973:2 to 1991:4 (the same sample considered by Mark (1995) consisting of 76 observations). Each experiment consist of 1000 Monte Carlo trials.

The exchange rate is modeled as a random walk and the fundamental as an $\operatorname{AR}(1)$ with persistence parameter equal to $.92 .^{3}$ This choice for the fundamental was made by fitting ARMA models to the actual U.S.-German data with the BIC selecting the lag orders. For each Monte Carlo dataset, the long-horizon regressions (equations 2) are run for $\mathrm{k}=1,4$, 8,12 and 16. The results for a sample size of 76 observations are presented in Table 1, panel A. Column 3 displays the median, 90th percentile and 95th percentile of the estimated slope coefficients across Monte Carlo trials for each horizon of interest. Despite the independence of the series, the median $\hat{\beta}$ rises with $\mathrm{k}$ to a maximum of .644 for $\mathrm{k}=16$. The associated naive OLS t-statistics displayed in column 4 also increase with the horizon. Columns 5 and 6

${ }^{3}$ In a second experiment, we generated both Monte Carlo series as random walks. The results were nearly identical. 
display t-statistics corrected for autocorrelation with a truncation lag of 20 and with the Andrews (1991) rule (labeled $\mathrm{t}(20)$ and $\mathrm{t}(\mathrm{A})$, respectively). Again, as the horizon increases so do median values of the slope coefficient's t-statistics. The right-shift of the empirical distribution of the t-statistics inflates the empirical critical values. For example, when $\mathrm{k}=16$ the one-sided empirical 95 th percentile for $t(\mathrm{~A})$ is 9.97 instead of 1.64, i.e., the corresponding critical value from a Gaussian distribution.

Column 8 of Table 1, panel A, displays the ratio of root-mean-squared error for outof-sample regression forecasts over root-mean-squared error implied by the random walk model. Thus, for values below 1 the regression appears to deliver more accurate forecasts than the benchmark random walk. As expected, the pattern is very similar to the results found by Mark (1995), Chinn and Meese (1995) and Bauer (1995). The median ratio declines from .968 to .796 , as the horizon of interest reaches $16 .^{4}$

Columns 9-10 display the Monte Carlo Diebold and Mariano (1995) statistics, again with either a truncation lag of 20 or using the Andrews (1991) rule (labeled DM(20) and $\mathrm{DM}(\mathrm{A})$ ). For both truncation rules, the values of the Diebold-Mariano statistics display a Ushape at each percentile. The median value using the Andrews rule is 1.213 for $\mathrm{k}=1$; it decreases somewhat for $\mathrm{k}=4,8$ only to increase to about 1.4 for $\mathrm{k}=12,16$.

These findings result from the combination of problems which afflict inference longhorizon regressions, when the two series fail to cointegrate. For example, although we argue

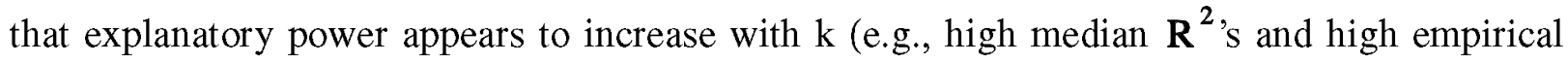
critical values of the t-statistics), there are sizeable distortions even for $k=1$. These biases arise because of the presence of stochastic and highly persistent regressors.

Such stark results obtain in sample sizes typical of available data. For any fixed and finite $\mathrm{k}$, a long-horizon regression will deliver consistent estimates as the sample size tends to

${ }^{4}$ At each Monte Carlo trial, the out-of-sample forecasting exercise precisely mimics the procedure in Mark (1995). For a sample size of 76, this results in 40 1-step ahead forecasts and 25 16-step ahead forecasts. 
infinity. ${ }^{5}$ In panel B of Table 1, we report the results of an identical simulation experiment with a sample size of 576. Now, the median $\hat{\beta}$ 's are all lower, but the bias is still sizable for large $\mathrm{k}$; for example, the median of $\hat{\beta}$ is .115 for $\mathrm{k}=16 . \mathrm{R}_{\mathrm{k}}^{2}$ are low and the ratios of RMSE of regression to random walk forecasts are very close to 1 for all horizons. However, the presence of small bias in the LS slope parameters introduces large distortions at all horizons in the empirical critical values of the t-statistics and the Diebold-Mariano statistics. These distortions do not seem to vanish quickly with the increase in the sample size.

It is important that finite sample inference from long-horizon regressions be assessed by means of empirical distributions of the statistics of interest simulated under the null of no statistical relationship between series. If standard Gaussian asymptotic theory is adopted, spurious evidence of predictability arises at practically all horizons and regardless of the length of the sample considered.

Lastly, we suggest that even the graphical evidence of predictability presented by Mark (1995) may be spurious. Figure 1 reproduces the actual k-period changes in the $\log$ dollar/DM rate against the regression forecast, displayed in Mark (1995). In the author's words, "these figures illustrate the striking improvement in fit that occurs as the forecast horizon is lengthened." In Figure 2 the actual k-period changes in the log dollar/DM rate is plotted against a regression forecast with simulated fundamental data. These plots also appear to suggest predictive accuracy for longer horizons. Since, in Figure 2 the exchange rate is obviously independent of the randomly generated fundamental, we conclude that even the graphical evidence from long-horizon regressions is misleading.

\section{Backward Regressions}

In this section, we adopt an approach to conducting long-horizon inference originally suggested by Jegadeesh (1991). This approach has been implemented by Hodrick (1992) to test for the presence of long-horizon predictability of stock returns, and recently by Mark and

${ }^{5}$ However, Richardson and Stock (1989) show that LS estimates of the slope of regressions (2) are inconsistent when the forecast horizon grows with the sample size (so that $\mathrm{k} / \mathrm{T} \rightarrow \delta$, a constant). 
Choi (1995) to test for the presence of long-horizon predictability of real exchange rates. Notice that equations 1 may be re-written as,

$$
\Delta \mathrm{s}_{\mathrm{t}+\mathrm{k}}+\Delta \mathrm{s}_{\mathrm{t}+\mathrm{k}-1}+\ldots+\Delta \mathrm{s}_{\mathrm{t}+1}=\alpha_{\mathrm{k}}+\beta_{\mathrm{k} \text { t }} \mathrm{z}_{\mathrm{k}, \mathrm{t}}
$$

The backward regression approach of Jegadeesh rearranges the implicit lag operator polynomial to the right hand side,

$$
\Delta \mathrm{s}_{\mathrm{t}+1}=\mathrm{a}_{\mathrm{k}}+\mathrm{b}_{\mathrm{k}}\left(\mathrm{z}_{\mathrm{t}}+\ldots+\mathrm{z}_{\mathrm{t}-(\mathrm{k}-1)}\right)+\mathrm{e}_{\mathrm{k}, \mathrm{t}}
$$

Under the null hypothesis of no predictive power, the $\hat{\beta}_{k}$ of equations (4) and $\hat{b}_{k}$ of (5) obey standard asymptotic theory for any fixed k. However, equations (5) are likely to exhibit better finite sample behavior because, under the null, the error term has no serial correlation. Notice that (4) and (5) are exactly equivalent for $\mathrm{k}=1$.

In order to verify that this procedure leads to more accurate rejection rates, we conducted a simulation experiment in which, again, the generated exchange rates and fundamentals are independent. ${ }^{6}$ The results are presented in Table 2.

Panel A of Table 2 contains the simulation results of the sampling performance of the backward regression (5) for a sample size of 76. Column 3 verifies that the $\hat{b}_{k}$ 's are small and do not increase with $\mathrm{k}$. The one-sided empirical critical values of the $\mathrm{t}(\mathrm{A})$-statistic for regression (5) remain constant for all k's (column 6). $\mathrm{R}_{\mathrm{k}}^{2}$ are consistently low across horizons (column 7). For completeness, panel B of Table 2 presents the results for sample size of 576.

\section{Long-run Exchange Rate Predictability}

${ }^{6}$ Hodrick (1992) analyzes the size distortions pertaining to coefficients estimated via equations (4) and (5). In contrast to this comment, Hodrick focuses only on large samples (much larger than the ones encountered in exchange rate studies). Moreover, the regressor of Hodrick's long-horizon equations, $z_{t}$ of (4), is not precisely an error-correction term. This implies, in particular, that the importance of the bias of the LS coefficients in Hodrick's longhorizon regressions may be somewhat different from the one arising in presence of exact error-correction terms. 
Given the ability of equation (5) to deliver more accurate finite-sample inference, we estimate equation (5) with 4 leading dollar exchange rates. The sample includes Germany, Canada, Japan and Switzerland, as in Mark (1995). The data are quarterly observations collected from the International Financial Statistics of the International Monetary Fund, from 1973:2 to 1994:1. A11 macroeconomic series are seasonally adjusted. Real income is real GNP for the U.S. and real GDP for other countries.

Table 3 displays the estimation results. At odds with evidence presented in Mark (1995), the $\hat{b}_{k}$ 's in column 2 do not increase with the horizon length. Indeed, they decrease with the horizon for the four exchange rates considered. Further, when using the critical values associated with the percentiles of the empirical distributions of $t(A)$ from the Monte Carlo (see Table 2, panel A), we cannot reject the null hypothesis of no predictability content of fundamental exchange rates for all currencies and horizons at the $90 \%$ confidence level. For example, at $\mathrm{k}=16$ the dollar/DM t(A) statistic is 2.511 which would exceed the standard normal $90 \%$ critical value of 1.208 . However, the tabulated statistics are biased upward so that the simulated $90 \%$ critical value is 2.553 . For the Canadian dollar and the yen, t-statistics are flat across horizons, while they appear to decrease for the Swiss franc.

$\mathrm{R}^{2}$, s increase with the horizon only for the dollar/DM rate. Indeed, the $\mathbf{R}^{2}$ for $k=12,16$ exceed the 95 th percentile of $R^{2}$ simulated under the null. Nevertheless, the $R^{2}$ reaches a maximum of only .129 as compared to .762 in Mark (1995).

Mark (1995) finds favorable evidence of the ability of the error-correction to forecast out-of-sample at horizons $\mathrm{k}=12,16$. Column 7, labeled OUT/RW, displays the regression mean-squared forecast errors over the random walk mean-squared forecast errors. For the Canadian dollar, yen and Swiss franc, these ratios are close to 1 at all horizons. For the Deutsche mark and the Swiss franc the ratio is significant at $k=8,12,16$, given the simulated 90\% critical values. However, the computed Diebold-Mariano statistics with the Andrews truncation rule, reported in column 9 of Table 3, are never larger than the 90th percentiles of the empirical distributions.

The estimation results of the backward regressions, when inference is made allowing for the presence of bias in estimated parameters and diagnostic statistics, suggest no longhorizon predictability via monetary fundamentals. These findings are at odds with the 
evidence reported by Mark (1995), Chinn and Meese (1995) and Bauer (1996) obtained using long-horizon regressions.

\section{Conclusion}

Applied economists must choose and interpret statistical methods with great care. The use of long-horizon regressions, in particular, may lead to spurious findings of predictive ability at long-horizons.

The results of a Monte Carlo study, in which two independent series are generated, confirm that applying long-horizon regressions to data which fail to cointegrate yields diagnostic statistics (e.g., t-statistics and Diebold-Mariano statistics) which display severe size distortions. A second simulation experiment suggests that the same diagnostic statistics generated from an alternative regression approach, due to Jegadeesh (1991), have better size properties.

Applying this methodology to historical data, we find neither in-sample nor out-ofsample evidence that fundamentals help to predict movements in major U.S. dollar exchange rates as the forecast horizon lengthens. 


\section{References}

Andrews, D.W.K. (1991), "Heteroskedasticity and Autocorrelation Consistent Covariance Matrix Estimation," Econometrica, 59, pp.817-58.

Basawa, L.V., A.K. Mallik, W.P. McCormick, J.H. Reeves, and R.L. Taylor (1991), "Bootstrapping Unstable First Order Autoregressive Processes," Annals of Statistics, 19, pp. 1098-1101.

Bauer, G. (1995), "The Foreign Exchange Risk Premium Over the Long Run," manuscript, Department of Finance, University of Pennsylvania.

Campbell, J.Y. and R.J. Shiller (1988), "Stock Prices, Earnings, and Expected Dividends," Journal of Finance, 43, pp. 661-76.

Chinn, M.D. and R.A. Meese (1995), "Banking on Currency Forecasts: How predictable is Change in Money ?" Journal of International Economics, 38, pp. 53-69.

Datta, S. (1995), "Limit Theory and Bootstrap for Explosive and Partially Explosive Autoregression," Stochastic Processes and their Applications, 57, pp. 285-304.

Diebold, F.X. (1991), Empirical Modeling of Exchange Rate Dynamics, New York: SpringerVerlag.

Diebold, F.X. and R.S. Mariano (1995), “Comparing Predictive Accuracy,” Journal of Business and Economic Statistics, 13, pp.253-262.

Diebold, F.X. and J.A. Nason (1990), "Non-parametric Exchange Rate Prediction ?" Journal of International Economics," 28, pp.315-32.

Fama, E. F. and K.R. French (1988), "Dividend Yields and Expected Stock Returns," Journal of Financial Economics, 22, pp. 3-25.

Granger, C.W.J and P. Newbold (1986), Forecasting Economic Time Series, San Diego: Academic Press.

Hodrick, R.J. (1992), "Dividend Yields and Expected Stock Returns: Alternative Procedures for Inference and Measurement," Review of Financial Studies, 5, pp.357-86.

Jegadeesh, N. (1991), "Seasonality in Stock Price Mean Reversion: Evidence from the U.S. and the U.K.," Journal of Finance, 46, pp. 1427-1444. 
MacDonald, R. and I.W. Marsh (1995), “On Cassellian PPP, Cointegration, and Exchange Rate Forecasting," Review of Economic Studies, forthcoming.

Mankiw, N.G. and M.D. Shapiro (1986), “Do We Reject Too Often?,” Economic Letters, 20, pp. 139-145.

Mark, N.C. (1995), "Exchange Rates and Fundamentals: Evidence on Long-Horizon Predictability," American Economic Review, 85, pp. 201-18.

Mark, N.C. and D. Choi, (1995) "Real Exchange Rate Prediction over Long Horizons," mimeo, Ohio State University.

Meese, R.A. and A.K. Rose (1991), "An Empirical Assessment of Nonlinearities in Models of Exchange Rate Determination", Review of Economic Studies, 55, pp. 703-708.

Nelson C.R. and M.J. Kim (1993), "Predictable Stock Returns: the Role of Small Sample Bias," Journal of Finance, 48, pp. 641-661.

Richardson, M. and J. Stock (1989), "Drawing Inferences from Statistics Based on Multi-Year Asset Returns," Journal of Financial Economics, 25, pp. 323-348.

Stambaugh, R.F. (1986), "Bias in Regression with Lagged Stochastic Regressors," Center for Research in Securities Prices Working Paper \#156. 
Table 1 - Long-Horizon Monte Carlo Estimates: Random Walks and Independent AR(1)

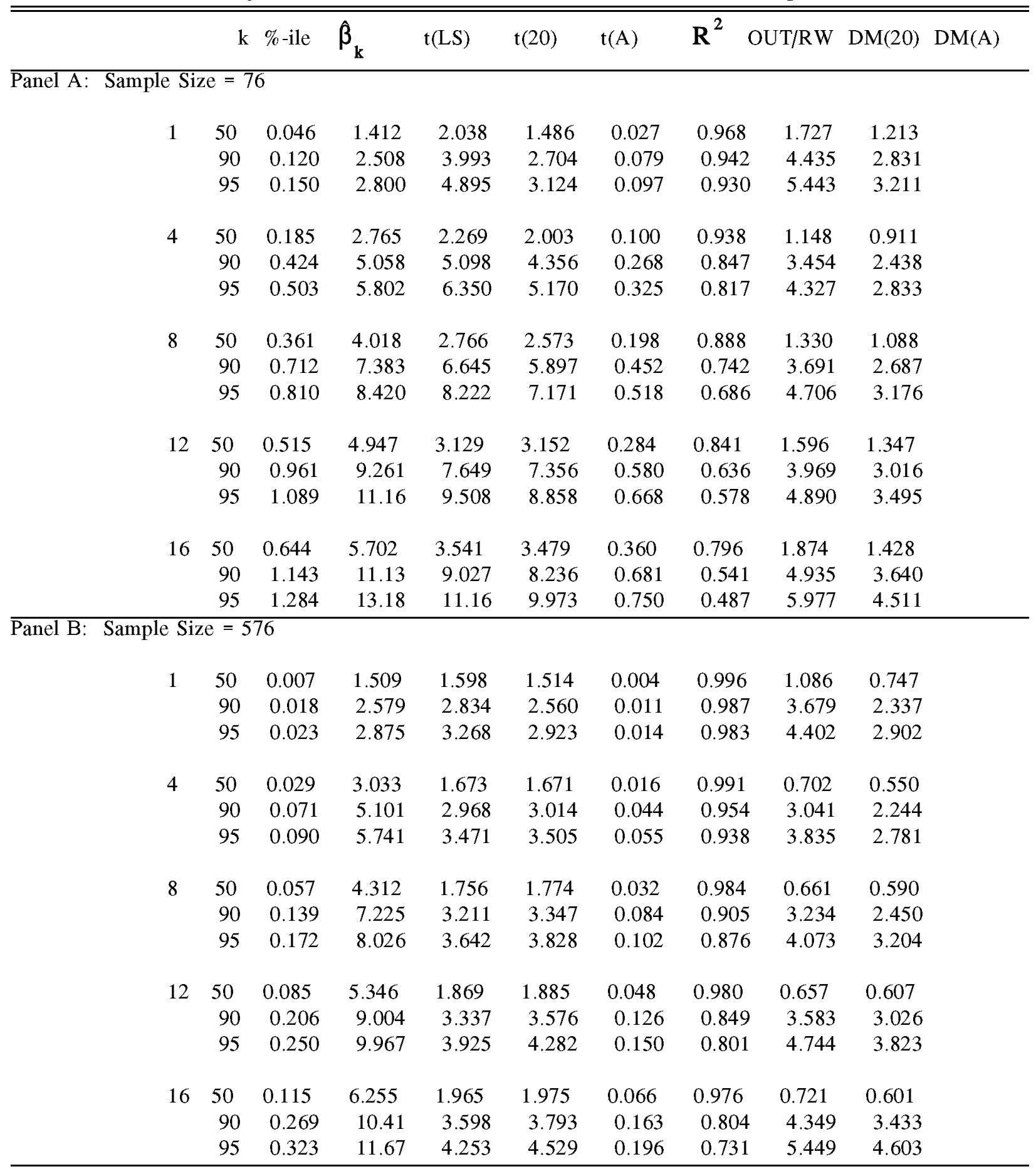

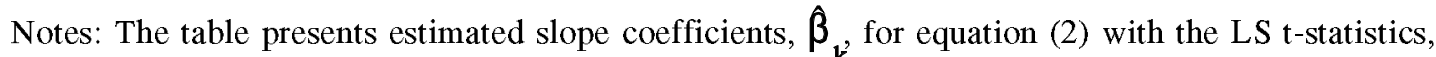
heteroskedasticity and autocorrelation-corrected t-statistics using a Bartlett kernel and a truncation lag of 20 and the Andrews (1991) rule, respectively, t(LS), t(20) and t(A). OUT/RW denotes the ratio of regression mean-squared out-of-sample forecast error to the random walk mean-squared out-of-sample forecast error. DM(20) and DM(A) denote the Diebold-Mariano statistics with a Bartlett kernel and truncation lags of 20 and truncation via the Andrews (1991) rule, respectively. 
Table 2 - Backward Regression Monte Carlo Estimates: Random Walks and Independent AR(1)

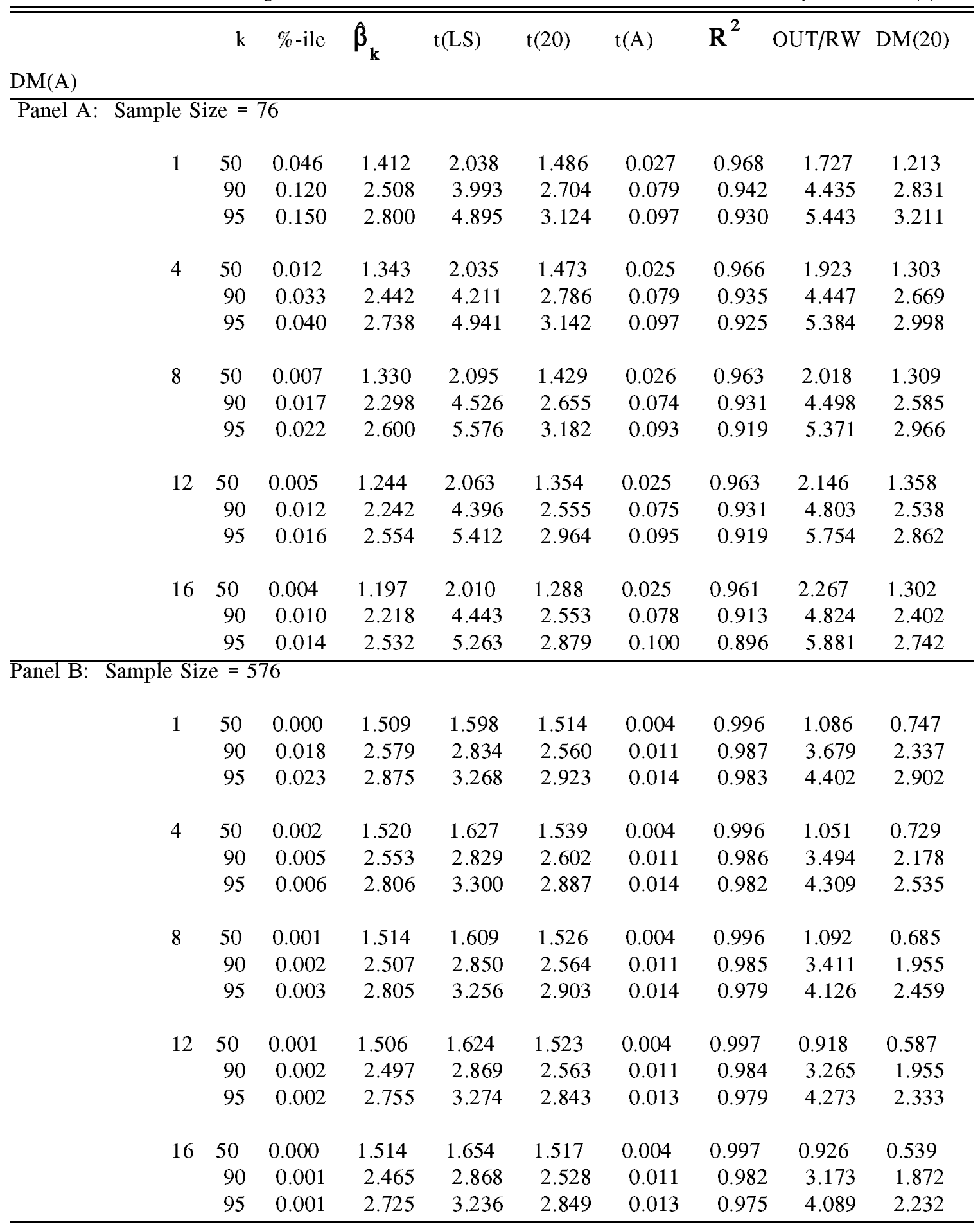

Notes: The table presents estimated slope coefficients, $\hat{b}_{k}$, for equation (5) with the LS t-statistics, heteroskedasticity and autocorrelation-corrected t-statistics using a Bartlett kernel and a truncation lag of 20 and the Andrews (1991) rule, respectively, $t(L S), t(20)$ and $t(A)$. OUT/RW denotes the ratio of regression mean-squared out-of-sample forecast error to the random walk mean-squared out-of-sample forecast error. DM(20) and DM(A) denote the Diebold-Mariano statistics with a Bartlett kernel and truncation lags of 20 and truncation via the Andrews (1991) rule, respectively. 
Table 3 - Backward Regression LS Estimates

\begin{tabular}{lcccccccc}
\hline \hline $\mathrm{k}$ & $\hat{b}_{\mathrm{k}}$ & $\mathrm{t}(\mathrm{LS})$ & $\mathrm{t}(20)$ & $\mathrm{t}(\mathrm{A})$ & $\mathrm{R}^{2}$ & $\mathrm{OUT} / \mathrm{RW} \mathrm{DM}(20)$ & $\mathrm{DM}(\mathrm{A})$ \\
\hline \multicolumn{2}{c}{ Detusche Mark } \\
1 & 0.042 & 1.493 & 2.168 & 1.233 & 0.030 & 0.964 & 1.085 & 0.878 \\
4 & 0.011 & 1.507 & 1.994 & 1.158 & 0.031 & 0.940 & 2.767 & 1.940 \\
8 & 0.009 & 2.140 & 2.453 & 1.565 & 0.065 & 0.921 & 1.493 & 1.267 \\
12 & 0.008 & 2.723 & 3.165 & 2.108 & 0.107 & 0.898 & 1.140 & 1.107 \\
16 & 0.008 & 2.931 & 2.998 & 2.511 & 0.129 & 0.901 & 0.817 & 0.879
\end{tabular}

\begin{tabular}{lllllllrr}
\multicolumn{2}{l}{ Canadian Dollar } & & & & & & & \\
1 & 0.063 & 1.508 & 1.636 & 1.199 & 0.030 & 1.008 & -0.239 & -0.186 \\
4 & 0.014 & 1.256 & 1.871 & 1.320 & 0.022 & 0.994 & 0.240 & 0.136 \\
8 & 0.008 & 1.296 & 2.069 & 1.482 & 0.025 & 1.015 & -0.386 & -0.202 \\
12 & 0.006 & 1.255 & 2.615 & 1.448 & 0.025 & 0.985 & 0.574 & 0.320 \\
16 & 0.005 & 1.112 & 2.495 & 1.379 & 0.021 & 0.961 & 2.643 & 1.614
\end{tabular}

\begin{tabular}{lrlllllll}
\multicolumn{2}{l}{ Japanese Yen } & & & & & & & \\
1 & 0.017 & 0.515 & 0.775 & 0.801 & 0.004 & 0.985 & 4.306 & 2.047 \\
4 & 0.002 & 0.261 & 0.442 & 0.432 & 0.001 & 0.988 & 3.902 & 2.102 \\
8 & 0.003 & 0.653 & 0.948 & 0.681 & 0.006 & 0.986 & 3.099 & 1.406 \\
12 & 0.003 & 0.733 & 1.187 & 0.708 & 0.009 & 0.981 & 2.502 & 1.629 \\
16 & 0.002 & 0.525 & 0.919 & 0.475 & 0.005 & 0.992 & 2.527 & 1.003
\end{tabular}

Swiss Franc

$\begin{array}{lllllllll}1 & 0.130 & 2.346 & 4.710 & 2.115 & 0.070 & 0.949 & 5.378 & 2.044 \\ 4 & 0.041 & 2.678 & 5.155 & 2.276 & 0.093 & 0.956 & 4.241 & 1.691 \\ 8 & 0.025 & 2.776 & 4.792 & 2.458 & 0.105 & 0.966 & 1.906 & 1.025 \\ 12 & 0.015 & 2.055 & 3.789 & 1.805 & 0.064 & 0.967 & 1.996 & 1.084 \\ 16 & 0.013 & 1.791 & 2.823 & 1.422 & 0.052 & 0.971 & 1.020 & 0.669\end{array}$

Notes: The data are from the International Financial Statistics of the International Monetary Fund, 1973:2 to 1994:1. The table reports estimated slope coefficients and diagnostic statistics for equation (5). 
Figure 1. Changes in the Log Dollar/Deutsche Mark Exchange Rate and Monetary Fundamentals.
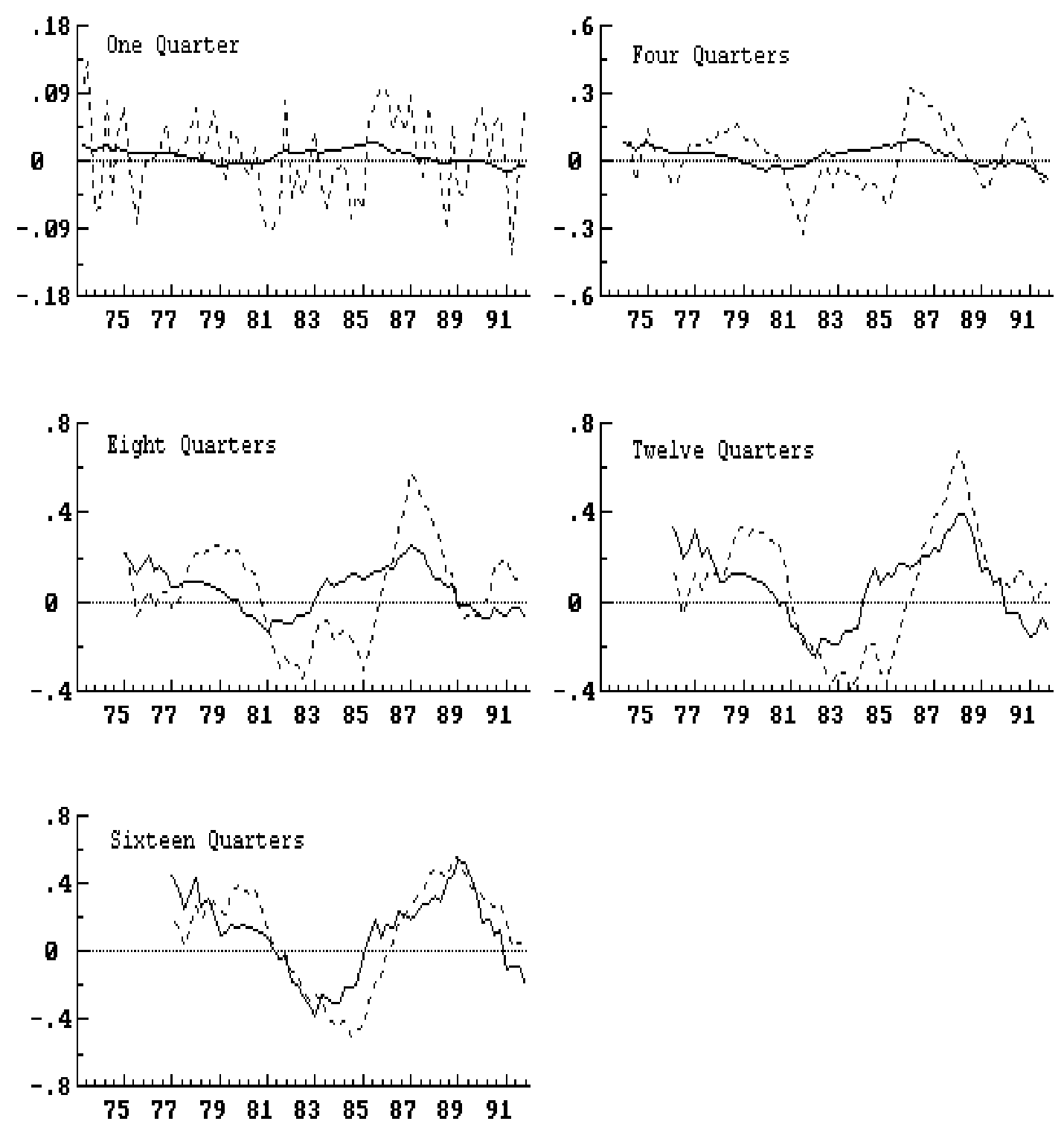

Notes to Figure: For each horizon of interest, $\mathrm{k}=1,4,8,12$ and 16, actual k-period changes in the log Dollar/DM rate are depicted as dashed lines. Solid lines indicate predicted k-period changes from long-horizon regressions on a monetary fundamental. Quarterly data from 1973:2 to 1991:4 were obtained from the International Financial Statistics of the International Monetary Fund. 
Figure 2. Changes in the Log Dollar/Deutsche Mark Exchange Rate and Simulated Fundamentals.
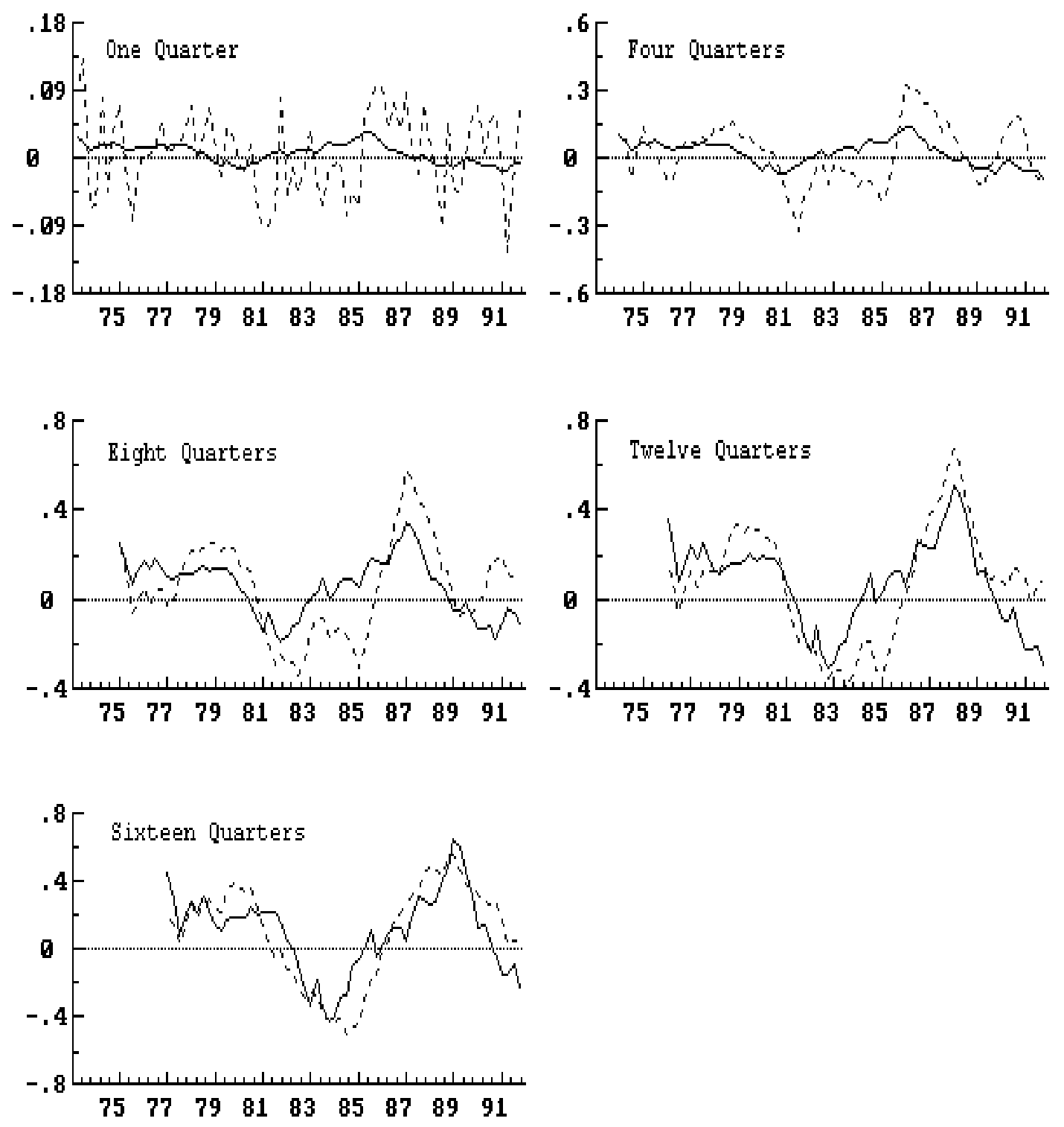

Notes to Figure: For each horizon of interest, $\mathrm{k}=1,4,8,12$ and 16, actual k-period changes in the log dollar/DM rate are depicted as dashed lines. Solid lines indicate predicted k-period changes from long-horizon regressions on independent random numbers. The random numbers are generated from a Gaussian AR(1) with persistence equal to .92 . 